\title{
9 Genetik: Die Entdeckung und Erforschung von FOXP2 als ein mahnendes Exempel
}

Given the chaotic state of the field, and the number of ever-proliferating theories, it is hardly surprising that researchers have tried to find some scientific Alexandrian sword that would quickly slice through the Gordian tangle of current confusion and provide a simple, straightforward solution.

Bickerton (2007: 523-524)

\subsection{Zum oft problematischen Fokus innerhalb einschlägiger Forschungsbemühungen}

Im Verlauf der vorliegenden Arbeit wurden der Umfang und die Vielseitigkeit der Forschungslandschaft zum Sprachursprung sowie der einschlägigen Literatur mitsamt ihren umfänglich widersprüchlichen Positionen bereits mehrmals erörtert. Bickerton folgert im zitierten Abschnitt in Anerkennung ebendieses Umstands ein Bedürfnis zur Klärung der Situation mittels möglichst eindeutiger Faktoren, jedoch bleibt zu unterstellen, dass nicht allein die konkrete, unsichere Situation eine derartige Tendenz der involvierten Autoren begründet, sondern eine tiefere Natur des Menschen. Der Fokus-Effekt bzw. die Fokus-Illusion beschreibt die generelle Tendenz, einen konkreten Aspekt oder wenige konkrete Faktoren einer eigentlich äußerst komplexen und schwer durchschaubaren Situation überzubetonen, um einen Effekt oder Zustand zu erklären respektive $\mathrm{zu}$ begründen. In den Worten von Kahneman et al. (2006, working paper): „When people consider the impact of any single factor [...] they are prone to exaggerate its importance; we refer to this tendency as the focusing illusion." Hierbei ist insbesondere die Reihenfolge, in der ein Gedankengang konstruiert wird, ausschlaggebend für das Eintreten des Fokus-Effekts. Um ein Beispiel zu nennen, seien Strack, Martin \& Schwarz (1988) knapp skizziert.

Diese konstruierten Experimente, in der Probanden zwei Fragen gestellt wurden - erstens die allgemeine Frage nach der Zufriedenheit mit dem eigenen Leben und zweitens eine konkretere Frage zur Zufriedenheit mit der aktuellen romantischen Situation. In dem Fall, dass die allgemeine Frage zuerst gestellt wurde, ergab sich keine statistisch signifikante Korrelation zwischen den Antworten auf die beiden Fragen. Die Antwort auf die erste Frage war hier also kein guter Prädiktor auf die Antwort der zweiten Frage. In dem anderen Fall, in welchem zunächst nach dem Liebesleben gefragt wurde und erst im Anschluss nach der allgemeinen Zufriedenheit, zeigte sich dagegen eine hohe Korrelation. 
Die Interpretation dieses Ergebnisses besagt, dass das allgemeinere Urteil eigentlich von einer Vielzahl von Faktoren abhängt, innerhalb derer das konkrete Merkmal nur einen kleinen Einfluss hat, dass aber durch die konkrete Frage der Fokus des jeweiligen Probanden auf ein einzelnes Merkmal gelenkt wurde, welches in Folge das allgemeinere Urteil maßgeblich - und durchaus unverhältnismäßig a.k.a. verfälschend - beeinflusst. Weitere Studien bestätigen diesen Effekt hinsichtlich verschiedener Situationen (vgl. bspw. Schkade \& Kahneman 1998 sowie Kahneman et al. 2006) und führen die Autoren zu einer warnenden Konklusion (Schkade \& Kahneman 1998: 345): „Nothing that you focus on will make as much difference as you think.“

Von besonderer Wichtigkeit ist nun die Feststellung, dass auch Wissenschaftler prinzipiell allen gängigen kognitiven Verzerrungen - einschließlich dem Fokus-Effekt - unterliegen und aus ebendiesem Grund strikte methodische Kontrollen einzuführen sowie einzuhalten haben. Die Bedeutung der vorgebrachten einleitenden Worte für den gegebenen Kontext der Sprachursprungsforschung konstituiert sich dabei auf zweierlei Weise. Erstens zeigt sich bereits der Weg der involvierten Autoren in dieses Forschungsfeld in ebenjenem Sinne als anfällig, da dieser gezwungenermaßen von der jeweiligen Expertise sowie den Konventionen der jeweiligen Einzelwissenschaft geprägt ist und damit in aller Regel zu einer implizit übermäßig fokussierten Perspektive führt. Zweitens tendieren darüber hinaus Neuentdeckungen dazu, in der Rezeption durch einschlägig aktive Autoren in problematischer Weise fokussiert und in diesem Zuge meist übergeneralisiert zu werden.

Dass in beiderlei Kontexten die Gefahr besteht, dass eine unangemessen einseitige Position eingenommen wird und dass dabei einer idealisierten, weil problematisch fokussierten, Argumentationslinie gefolgt wird, muss als gegeben hingenommen werden. Ein besonders aussagekräftiges Beispiel für ein derartiges Vorgehen sowie für eine problematische Idealisierung und eine defizitäre Übergeneralisierung stellt dabei die Forschungsgeschichte zum FOXP2Gen dar. Im Folgenden soll diese also knapp skizziert und in einen angemessenen Kontext gesetzt werden, um als ein mahnendes und lehrreiches Exempel zu dienen. Im Anschluss sollen weitere knappe Erläuterungen zu populationsgenetischen Erkenntnissen sowie deren problematischer Rezeption in der einschlägigen Literatur das vorliegende Kapitel abrunden.

\subsection{Die Entdeckung und anfängliche Erforschung von FOXP2}

Hurst et al. (1990), Gopnik (1990a und 1990b) sowie Gopnik \& Crago (1991) lieferten die ersten Beschreibungen eines erweiterten Familienkreises aus London, 
welcher anscheinend von einer dominant vererbten Sprachstörung betroffen war. Diagnostiziert wurden 16 Individuen über drei Generationen, wobei sich zwar lediglich geringe Defizite im Sprachverständnis fanden, jedoch umfangreiche Einschränkungen in der Effizienz der Sprachproduktion gepaart mit einigen grammatischen Unfähigkeiten - unter anderem die scheinbar systematische Unfähigkeit, morphologische Paradigmen $\mathrm{zu}$ erlernen, sodass bspw. der sPlural des Englischen nicht frei angewandt werden konnte, sondern lexikalisch erlernt werden musste. Die Interpretation dieser ersten Untersuchungen bestand mit überwältigender Einstimmigkeit in dem Postulat eines unterliegenden Grammatik-Gens. Gopnik (1990a: 715) beispielsweise schloss: „Because the deficits are apparent in all aspects of language, their roots probably lie in the underlying grammar rather than in a peripheral processing system.“ sowie „The distribution of dysphasia in this one large family suggests that it may be due to one dominant gene[.]“

Dieses erste Bild erweiterte sich in den nachfolgenden Jahren. VarghaKadem et al. (1995) beispielsweise beschreiben die Störungen als inkompatibel mit der Interpretation eines einfachen Grammatik-Gens, sondern attestieren umfangreiche Einschränkungen in mehreren kognitiven und physiologischen Bereichen. Auch betonen sie die variable Schwere der Einschränkungen innerhalb der KE-Familie, wie sie innerhalb der Literatur allgemein genannt wird. Jedoch auch im Kontext dieses umfangreichen Phänotyps mit Defiziten in Intelligenz, Artikulation und generell der Gesichtsmuskulatur seien die linguistischen Schwierigkeiten laut Vargha-Khadem et al. (1995) noch immer ein prominenter Teil des Gesamtbilds und böten damit potenziell Einblicke in die Grundlagen des menschlichen Sprach- und Sprechvermögens. Auch Fisher et al. (1998), deren primärer Beitrag durch eine vage Lokalisation des Gens auf Chromosom 7 und damit die Bestätigung einer dominanten Vererbung gegeben ist, betonen die sprachlichen Implikationen. Zwar sei laut ihnen kein simples Grammatik-Gen gegeben, jedoch sei ,virtually every aspect of grammar and of language affected“ (Fisher et al. 1998: 168), womit dort die Implikation eines scheinbaren Sprachgens weiterhin gegeben ist.

Eine Identifikation des Gens gelang schließlich Lai et al. (2001) und obwohl deren Urteil adäquat vorsichtig formuliert war („Our findings suggest that FOXP2 is involved in the developmental process that culminates in speech and language“, Seite 519), so hielt sich im populärwissenschaftlichen Bewusstsein weiterhin das Bild eines Grammatik- bzw. Sprach-Gens. Enard et al. (2002) erweiterten den einschlägigen Forschungsstand um den genetischen Vergleich mit dem nächsten rezenten Verwandten des Menschen und stellten trotz der Größe des Gens von etwa 600.000 Basenpaaren lediglich zwei Substitutionen 
fest. Zudem versuchten sich die Autoren an einer Einschätzung der zeitlichen Tiefe dieser Veränderung und vermuteten diese im Kontext eines 95\%Konfidenzintervalls bei weniger als 120.000 Jahren. Daraus schlossen sie, dass das Aufkommen des anatomisch modernen Menschen mit ebendieser Mutation und der Ausbildung einer sprachlichen Befähigung zusammengefallen sein müsse und setzten diese Feststellung in den Kontext der damals vorherrschenden Lehrmeinung der Überlegenheit des anatomisch modernen Menschen gegenüber dem Neandertaler (Enard et al. 2002: 871): „[O]ur method suggests that the fixation occured during the last 200,000 years of human history, that is, concomitant with or subsequent to the emergence of anatomically modern humans.“ sowie „This is compatible with a model in which the expansion of modern humans was driven by the appearance of a more-proficient spoken language.“

\subsection{Klärendes zur Genetik und zu funktionalen Implikationen von FOXP2}

Bevor der weitere Forschungsverlauf skizziert werden soll, muss noch knapp geklärt werden, weshalb und in welchem Umfang die starke Konservation des Gens von Bedeutung ist. Entgegen der populären Vorstellung zum Konzept der Mutation treten Mutationen in jeder Generation und in jedem Individuum auf, betreffen dabei jedoch allein statistisch die wesentlich umfangreichen Bereiche nicht-kodierender DNA und stellen damit „stumme“441 Mutationen ohne positive oder negative Auswirkungen dar, sondern erhöhen schlicht in einer Weise die genetische Variabilität einer Population (vgl. hierzu sowie zum Nachfolgenden bspw. Bohidar 2015 ab Seite 299). ${ }^{42}$

Auch in dem Fall, dass eine Mutation eine regulierende oder kodierende Region betrifft, besteht die Möglichkeit einer neutralen Substitution, jedoch entsteht hier oftmals stattdessen eine Beeinflussung bzw. auch Einschränkung ebenjener Funktion und lediglich äußerst selten stellt sich eine funktional positive Änderung des Genotyps ein. Regulierende Sequenzen zeigen hier mitunter

41 Der Begriff der stummen Mutation hat in der Genetik eine streng definierte Bedeutung. An gegebener Stelle wird der Begriff in einem erweiterten, vagen Sinn verwendet, um dem einschlägigen Laien eine hilfreiche Metapher anzubieten. Die „Stummheit“ soll veranschaulichen, dass derartige Mutationen die Funktion eines gegebenen Genoms nicht beeinflussen.

42 Wenn hier sowie im Weiteren von „Mutation“ gesprochen wird, so ist an gegebener Stelle eine vererbliche Keimbahnmutation gemeint und nicht eine somatische Mutation, da im gegebenen evolutionsbiologischen Kontext lediglich erstere Mutationsform von Bedeutung ist. 
eine nennenswerte Flexbilität in ihrem Verhältnis zwischen Struktur und Expression. Die Wahrscheinlichkeit dazu, ob sich Varianten letztendlich in einer Population etablieren können und in welchem Umfang dies geschieht, variiert je nach Bedeutung des Gens und Einfluss der Mutation sowie nicht zuletzt aufgrund statistischer Zufälligkeit. Insbesondere im Fall von funktional problematischen Mutationen, aber auch bei neutralen Mutationen auf höchst stabilen Genen, kann sich die entstandene genetische Variante aufgrund populationsgenetischer Prinzipien üblicherweise nicht in einer Population halten, sondern wird im Kontext einer negativen Selektion aus dem Genpool der Population bereinigt. Bei neutralen Varianten und wenn weniger stabile Gene betroffen sind können statistische Effekte auch dazu führen, dass sich Varianten innerhalb einer Population als Teil eines Gendrifts zunehmend etablieren, durchsetzen dabei jedoch oft nicht die gesamte Population. Zuletzt sind es freilich die äußerst seltenen positiven Mutationen, welche sich durch positive Selektion erleichtert innerhalb einer Population (und dabei ggf. sogar vollständig) verbreiten können.

Die äußerst hohe Stabilität, d.h. Homogenität innerhalb menschlicher Populationen aufgrund Freiheit von durch Mutationen entstandenen Varianten, von FOXP2 in einer evolutionären Perspektive zum Trotz der Größe des Gens zeugt dabei von einer entsprechend großen Wichtigkeit sowie einem äußerst starken Selektionsdruck auf die Stabilität desselben, sodass abweichende Formen durch negative Selektion rigoros aussortiert werden. Zusätzlich spricht der Umstand jener zwei gegenüber dem Schimpansen in der Gesamtpopulation des Menschen ubiquitär vorhandenen Substitutionen für eine außerordentliche Nützlichkeit derselben im Kontext derjenigen selektiven Einflüsse, welche auf relevante frühmenschliche Arten eingewirkt haben. Anders kann das dortige Aussetzen der ansonsten rigorosen negativen Selektion sowie die Ubiquität der modernen Variante nur schwer erklärt werden. Im Kontext des bereits zu FOXP2 Erläuterten liegt die naheliegende Unterstellung sicherlich in einem starken Selektionsdruck zum artikulierten Sprechen bzw. zu einem Sprachvermögen. Eine wichtige Feststellung findet sich also in der immensen funktionalen Bedeutung der beiden Substitutionen im Kontext ebenjenes phylogenetischen Spracherwerbs.

\subsection{Die weitere Erforschung von FOXP2 und Probleme der interdisziplinären Rezeption}

Jene Interpretation einer evolutionären Etablierung von Sprachbefähigung im anatomisch modernen Menschen durch eine FOXP2-Mutation, welche wie zuvor 
erwähnt bereits bei Enard et al. (2002) knapp skizziert wurde, erfuhr eine Popularisierung innerhalb der einschlägigen Literatur unter anderem durch Balter (2002) und fand in den nachfolgenden Jahren umfangreich Einzug in Veröffentlichungen der Sprachursprungsforschung. Insbesondere einschlägige Argumentationslinien, welche einen diskontinuierlichen Ursprung von Sprache zu attestieren suchten bzw. welche einen gestischen Sprachursprung vertraten, inkorporierten diese Interpretation einer jungen und äußerst bedeutsamen Mutation der modernen FOXP2-Variante. Für Erstere fand sich freilich ein dankbares genetisches Argument für eine harte Grenze zwischen Sprachlosigkeit und Sprachvermögen, während für Letztere eine junge Oralität die Implikation $\mathrm{zu}$ beinhalten schien, dass die phylogenetische Sprachentwicklung zunächst gestisch geschehen sein müsste. So heißt es beispielsweise bei Tomasello (2008: 235-236) sehr prägnant: „It is difficult to imagine any function other than articulate speech, as used in modern languages, for the incredibly fine-grained motor control that this gene seems to enable.“

Im Kontext dieser argumentativen Manie rund um FOXP2 - sowie in vergleichbarer Weise zu anderen zu jener Zeit entdeckten bzw. näher untersuchten Phänomenen wie den Spiegelneuronen - warnten andere Autoren jedoch vor einer verfrühten Überbetonung solcher vermeintlich praktischen und simplen Erklärungsansätzen. Bickerton (2007: 524-525) beispielsweise mahnt wie folgt zur Vorsicht: „[T]ake care, it's a minefield out there, strewn with explosive charges of little-known fact ready to blow up the fanciest new theory. "Die Adäquatheit ebendieser Warnung zeigte sich im Verlauf der weiterführenden einschlägigen Forschung. Zunächst schwächte sich die direkte Assoziation von FOXP2 mit sprachlicher Befähigung, denn zunehmend zeigte sich die umfassende Implikation des Gens in einer Reihe von regulativen Prozessen - unter anderem bei der Plastizität der ontogenetischen neuronalen Entwicklung (Haesler et al. 2004, Scharff \& Haesler 2005 sowie Fisher \& Scharff 2009) und bei so fernen physiologischen Funktionen wie in der Ausbildung von Lunge und Magen unter Vorhandensein einer komplexen gegenseitigen Regulation mit verschiedenen anderen Genen (vgl. bspw. Shu et al. 2007; siehe für knappe Zusammenfassungen der Forschung bis zu jenen Punkten Preuss 2012 sowie Nudel \& Newbury 2013).

In anderen Worten spielt FOXP2 vermutlich eine gewichtige Rolle in der Neurogenese, also der Ausbildung der neuronalen Infrastruktur, welche Sprache begünstigend oder ermöglichend zugrunde liegt, jedoch besteht ebendiese Rolle in einer indirekten statt in einer direkten Natur und der genaue kausale Beitrag zu einer sprachlichen Befähigung ist schwer einzuschätzen. In diesem Sinne zeugen genetische Veränderungen im Verlauf der Menschwerdung all- 
gemein und einschließlich FOXP2 vermutlich nicht von einschneidenden Revolutionen, welche praktischerweise die Gesamtsituation im Sinne eines harten Bruchs aufklären. Stattdessen legen derartige Veränderungen vielmehr ein vormals bereits bestehendes - wenn auch gegebenenfalls archaisches, prototypisches - Verhalten nahe, aus welchem ein Selektionsdruck folgte, der genetische Veränderungen hin zu einer größeren oder auch nur besser verfügbaren Leistungsfähigkeit in dem selektierten Leistungsmerkmal begünstigte. Die jeweilige genetische Änderung unterstützt bzw. vereinfacht damit den Umgang des Organismus mit den Anforderungen seines Umfelds, anstatt eine Domäne der Befähigung de novo entstehen zu lassen. Dies soll eine Rolle im phylogenetischen Spracherwerb nicht verneinen, jedoch ist mit Vorsicht und analytischer Nuance auf die einschlägigen Erkenntnisse einzugehen - eine Vorgabe, welche in der einschlägigen Forschung umfassend nicht erfüllt wird.

Weiterhin - und hinsichtlich jener Argumentation in nochmals konkreterem Maße problematisch - zeigen Krause et al. (2007), Reich et al. (2010) und Paixáo-Côrtez et al. (2012), dass nicht nur der anatomisch moderne Mensch, sondern auch die Schwesterngruppen des Neandertalers sowie der DenisovaMenschen die moderne Variante des FOXP2-Gens ebenfalls besaßen. Daraus folgt freilich eine völlige Umkehrung des vorgenannten Arguments zum späten artikulierten Sprechen, denn unter der Annahme der Zentralität des Gens für jene Befähigung würde sich ebendiese zeitlich zum gemeinsamen Vorfahren jener Menschengruppen verschieben. Weiter gestärkt wird dieses Bild durch Atkinson et al. (2018), welche zeigen, dass FOXP2 innerhalb der Evolutionsgeschichte von $H$. sapiens keine positive Selektion mehr erfuhr. In anderen Worten: Funktional war FOXP2 zu diesem Zeitpunkt - und daher potenziell auch im Neandertaler - bereits quasi modern.

\subsection{Implikationen und Schlussfolgerungen für die Sprachursprungsforschung}

Diese Feststellungen zeigen sich freilich als gegenseitig stützend mit den Ausführungen des vorigen Abschnittes zur jüngeren paläoanthropologischen Datenlage, jedoch ist die funktionale und regulative Komplexität von FOXP2 bis heute noch nicht umfänglich erschlossen, sodass von allzu starken Schlussfolgerungen abgesehen werden sollte. An gegebener Stelle sei also zu vermeiden, in eine ähnlich problematische Spekulation zu verfallen wie bei früheren Autoren gegeben war. Dennoch verbleiben die gemachten vorbehaltlichen Schlussfolgerungen als bedeutsame Beobachtungen von nennenswerter Relevanz für die Gesamtdiskussion. Weiterhin konnte im vorliegenden Abschnitt veran- 
schaulicht werden, in welcher Weise eine angemessene Zurückhaltung in der Bewertung provisorischer Forschungsergebnisse notwendig zu sein scheint, um problematischen Vorgehensweisen wie Übergeneralisierung vorzubeugen. Methodische Stringenz in der Rezeption von Literatur und der verfügbaren Datenlage vermag es, gegenüber derartigen Problemen sowie hinsichtlich des FokusEffekts eine kontrollierende Funktion innezuhaben.

Im Sinne des vorgestellten Methodenkatalogs zeigt sich innerhalb der Literaturrezeption wiederum eine undurchsichtige Situation hinsichtlich M1, jedoch warnt M2 vor jenen vorschnellen, idealisierenden Interpretationen eines konkreten „Grammatik-Gens“ und M4 verzeichnet jene spekulativen Ableitungen aus den genetischen Erkenntnissen als risikobehaftet. M3 gestaltet sich hier wenigstens teilweise als nicht ausreichend aussagekräftig, da aufgrund der vorliegenden Situation eines Forschungsfeldes, welches sich zur Jahrtausendwende noch in methodischen Frühstadien befand, auch die Expertenposition von Lai et al. (2001) noch $\mathrm{zu}$ problematischen Schlussfolgerungen führte. In diesem Sinne warnt M5 vor einer Überbewertung ebenjener Ergebnisse, denn sie waren $\mathrm{zu}$ jenem Zeitpunkt noch nicht ausreichend repliziert bzw. trianguliert. Insbesondere M8 und M9 fundieren schließlich eine angemessene Perspektive zu den verfügbaren Ergebnissen. Zu Zeiten jener spekulativen Fehlschlüsse wäre auch laut diesen Prinzipien noch zur Vorsicht geraten worden erst recht unter Beachtung des Prinzips M12. Die Rezeption innerhalb der Sprachursprungsforschung zeugt in jedem Fall von einem umfangreichen Scheitern metawissenschaftlicher Sensibilitäten und steht damit im gegebenen Kontext als ein warnendes Exempel.

\subsection{Zusätzliches zu populationsgenetischen Erkenntnissen}

Freilich bestehen problematische Argumentationslinien auch jenseits der bereits beschriebenen Forschungsgeschichte des FOXP2-Gens hinsichtlich der Genetik innerhalb der Sprachursprungsforschung - und so sollen populationsgenetische Aussagen in Berwick \& Chomsky (2017: 167-168) in knappster Weise kommentiert werden, um dies weiter zu veranschaulichen. Jene bringen Studien vor, welche den genetischen Beitrag von Neandertalern und DenisovaMenschen zu nicht-afrikanischen modernen Menschen untersuchen, und fokussieren insbesondere das Ergebnis, dass viele Regionen des modernen Genoms von Beiträgen jener archaischen Menschenarten bereinigt sind (bspw. Sankararaman et al. 2016). Darauf aufbauend argumentieren sie dafür, dass diese Selektion gegen die archaischen Genotypen als Zeichen dafür zu sehen ist, „that there may have been subtle genomic differences that accumulated on the 
Homo sapiens lineage, distinct from the Neandertal/Denisovans, leading to a qualitatively different phenotype“ (Berwick \& Chomsky 2017: 168). In anderen Worten sei aus der negativen Selektion gegen archaische Varianten im Nachgang zur genetischen Durchmischung zwischen dem Neandertaler und dem modernen Menschen ein Hinweis darauf zu folgern, dass der Genotyp respektive der damit verbundene Phänotyp des modernen Menschen qualitativ überlegen gewesen sei. Die Bereinigung des modernen Genoms von archaischen Varianten sei in diesem Sinne ein Hinweis darauf, dass beim Neandertaler kein Sprachvermögen inferiert werden kann.

An dieser Stelle ist nochmals auf die vorigen Erläuterungen zu verweisen, laut denen kodierende und mitunter regulierende Bereiche des Genoms, welche als funktional bedeutend anzusehen sind, sich als höchst stabil erweisen, da sie beständig positiv selektiert werden, um ihre Funktionalität angemessen aufrechtzuerhalten. Weiterhin ist wiederum darauf hinzuweisen, dass der anatomisch moderne Mensch im Zeitraum der genetischen Durchmischung mit den archaischen menschlichen Taxa in demographischer Hinsicht die stark dominierende Population darstellte und nach dem Verschwinden derselben freilich die einzige verbliebene Population darstellte. In einer derartigen Situation und hier liegt das primäre Missverständnis durch Berwick \& Chomsky (2017) reichen Prinzipien der Populationsgenetik aus, um ein Szenario dazu zu bieten, weshalb archaische Varianten in der Entwicklung zum modernen Genom bereinigt wurden.

Statistische Prinzipien des Gendrifts erklären in diesem Sinne ausreichend, wie die Neandertaler-DNA unabhängig von ihrer „phänotypischen qualitativen Wertigkeit“ in der Populationsdynamik des modernen Menschen sukzessive verloren gehen (vgl. Juric, Aeschbacher \& Coop 2016 und Petr et al. 2019). Allein diejenigen Beiträge des Neandertalers respektive des Denisova-Menschen oder anderer archaischer Populationen, welche einen klaren Vorteil für die Population des anatomisch modernen Menschen im Wechselspiel zwischen der vorhandenen ökologischen Situation und dessen Phänotyp zu bieten vermochten und zugleich kompatibel mit dessen genomischen funktionalen Geflecht waren, konnten sich dauerhaft im Genom des nicht-afrikanischen $H$. sapiens fixieren anstatt in der weiteren Populationsdynamik weitgehend aus dem Genpool verdrängt $\mathrm{zu}$ werden. Dies begründet sich darin, dass die genetischen Varianten des anatomisch modernen Menschen aufgrund dessen größeren und alleinig fortbestehenden Populationen um ein vielfaches zahlreicher im Genpool vorkommen. Damit führen allein statistische Prinzipien zu einer allmählichen Bereinigung von Varianten des Neandertalers, ohne dass dessen Varianten als funktional unterlegen deklariert werden müssen. In der Tat sind es allein funk- 
tional überlegene Varianten, welche diesem Prozess standhalten und sich fest im Genom des modernen Menschen etablieren konnten.

Dem ist freilich nicht $\mathrm{zu}$ folgern, dass es keine funktionalen Inkompatibilitäten zwischen dem Neandertaler und dem anatomisch modernen Menschen gegeben habe. Ebenso bleibt es durchaus im Bereich des Möglichen, dass eine funktional begründete negative Selektion gegen Abschnitte des archaischen Genoms stattgefunden hat. Die verfügbaren Daten lassen hierzu schlicht (noch) keine angemessenen Aussagen zu. Festzustellen ist jedoch, dass Berwick \& Chomsky (2017) eine unangemessen einseitige Auslegung der Datenlage vorlegen und problematische Schlussfolgerungen aus dieser übermäßig starren Interpretation ziehen. Im Grunde liegt der Fehlschluss in der Umkehrung des Modus ponens: Wenn der Neandertaler dem anatomisch modernen Menschen in einer Weise unterlegen wäre, welche jene Autoren unterstellen, dann wäre durchaus das genetische Muster festzustellen, welches dokumentiert wurde. Jedoch kann aus der Feststellung ebenjenes Musters nicht im Umkehrschluss auf die Unterlegenheit des Neandertalers gefolgert werden, denn es existieren auch alternative Mechanismen bzw. Szenarien, welche das gefundene Muster hinreichend erklären können.

Damit sei das zentrale Anliegen des vorliegenden Abschnitts - also das Aufzeigen von Missverständnissen und problematischen Schlussfolgerungen als abgeschlossen zu gelten. Die vorliegende Kritik begrenzt sich auf eine knappe Erläuterung, um weiteren Unterkapiteln ausreichend Raum zu bieten und damit in letzter Konsequenz ein thematisch breites Vorgehen zu ermöglichen. Dies ist notwendig, um die Leistungsfähigkeit des methodischen Programms der vorliegenden Arbeit umfassend demonstrieren zu können. Als ein dieses Unterkapitel abschließender Gedanke sei lediglich noch einmal darauf verwiesen, dass ein gewissenhaftes metawissenschaftliches Vorgehen im Kontext einer angemessen vielseitigen Rezeption der Literatur Problemen dieser Art vorzubeugen vermag - und insbesondere im Kontext des vorliegenden höchst interdisziplinären Forschungsfeldes ist freilich mit besonderer Vorsicht sowie Sorgfalt vorzugehen. 